(n)

trobertivier Journal of Nonlinear Mathematical Physics

\title{
Differential Equations Invariant Under Conditional Symmetries
}

Decio Levi, Miguel A. Rodríguez, Zora Thomova

To cite this article: Decio Levi, Miguel A. Rodríguez, Zora Thomova (2019) Differential Equations Invariant Under Conditional Symmetries, Journal of Nonlinear Mathematical Physics 26:2, 281-293, DOI: https://doi.org/10.1080/14029251.2019.1591731

To link to this article: https://doi.org/10.1080/14029251.2019.1591731

Published online: 04 January 2021 


\title{
Differential Equations Invariant Under Conditional Symmetries
}

\author{
Decio Levi \\ INFN, Sezione Roma Tre, Via della Vasca Navale 84 \\ 00146 Roma, Italy \\ levi@ roma.infn.it \\ Miguel A. Rodríguez \\ Dept. de Física Teórica, Pza. de las Ciencias 1, Universidad Complutense de Madrid \\ 28040 Madrid, Spain \\ rodrigue@ucm.es \\ Zora Thomova \\ SUNY Polytechnic Institute, 100 Seymour Road, Utica, NY 13502, USA \\ Zora.Thomova@sunypoly.edu
}

Received 18 April 2018

Accepted 18 December 2018

\begin{abstract}
Nonlinear PDE's having given conditional symmetries are constructed. They are obtained starting from the invariants of the conditional symmetry generator and imposing the extra condition given by the characteristic of the symmetry. Series of examples starting from the Boussinesq and including non-autonomous Korteweg-de Vries like equations are given to show and clarify the methodology introduced.
\end{abstract}

Keywords: Lie symmetries; partial differential equations; conditional symmetries

\section{Introduction}

As Galileo Galilei said in Il Saggiatore (1623) [21], our world is described in mathematical formulas and it is up to us to comprehend it. This was the starting point of the scientific revolution which goes on up to nowadays and gave us the present world technology, i.e. cellular phones, lasers, computers, nuclear resonance imaging, etc.

Our capability of solving complicated physical problems described by mathematical formulas (say equations) is based on the existence of symmetries, i.e. transformations which leave the equations invariant. Towards the end of the nineteenth century, Sophus Lie introduced the notion of Lie group of symmetries in order to study the solutions of differential equations. He showed the following main property: if an equation is invariant under a one-parameter Lie group of point transformations then we can reduce the equation and possibly construct an invariant solution. This observation unified and extended the available integration techniques such as separation of variables or integrating factors. Roughly speaking, Lie point symmetries are a local group of transformations which map every solution of the system into another solution of the same system. In other words, it maps the solution set of the equation into itself. 
A partial differential equation (PDE) $\mathscr{E}=0$ is invariant under a continuous group of Lie point transformations if the corresponding infinitesimal symmetry generator $\hat{X}$ satisfy

$$
\left.\operatorname{pr} \hat{X} \mathscr{E}\right|_{\mathscr{E}=0}=0
$$

where by the symbol pr we mean the prolongation of the infinitesimal generator to all derivatives appearing in the equation $\mathscr{E}=0$. In particular, if we consider a second order PDE in $\mathbb{R}^{2}$ of independent variables $x$ and $y$ and dependent variable $u(x, y)$,

$$
\mathscr{E}=\mathscr{E}\left(x, y, u, u_{x}, u_{y}, u_{x x}, u_{x y}, u_{y y}\right)=0,
$$

(where the subscripts denote partial derivatives) the infinitesimal generator will be given by

$$
\hat{X}=\xi(x, y, u) \partial_{x}+\eta(x, y, u) \partial_{y}+\phi(x, y, u) \partial_{u},
$$

where $\xi, \eta$ and $\phi$ are functions of their arguments to be determined by solving (1.1). The prolongation of $\hat{X}$ is given by

$$
\begin{aligned}
\operatorname{pr} \hat{X}=\hat{X} & +\phi^{(1, x)}\left(x, y, u, u_{x}, u_{y}\right) \partial_{u_{x}}+\phi^{(1, y)}\left(x, y, u, u_{x}, u_{y}\right) \partial_{u_{y}}+ \\
& +\phi^{(2, x x)}\left(x, y, u, u_{x}, u_{y}, u_{x x}, u_{x y}, u_{y y}\right) \partial_{u_{x x}}+ \\
& +\phi^{(2, x y)}\left(x, y, u, u_{x}, u_{y}, u_{x x}, u_{x y}, u_{y y}\right) \partial_{u_{x y}}+ \\
& +\phi^{(2, y y)}\left(x, y, u, u_{x}, u_{y}, u_{x x}, u_{x y}, u_{y y}\right) \partial_{u_{y y}},
\end{aligned}
$$

where the functions $\phi^{(1, x)}, \phi^{(1, y)}$ and $\phi^{(2, x x)}, \phi^{(2, x y)}, \phi^{(2, y y)}$ are algorithmically derived in terms of $\xi, \eta$ and $\phi$. See, for example, the references $[7,36]$ for this construction.

Given an infinitesimal generator of a symmetry $\hat{X}$ a function $\mathscr{I}$ is an invariant if it is such that

$$
\operatorname{pr} \hat{X} \mathscr{I}=0 .
$$

Eq. (1.5) is a first order PDE which can be solved on the characteristic and provide the set of invariants and differential invariants $\mathscr{I}_{j}, j=0,1, \ldots$ depending on $x, y, u$ and its partial derivatives up to the second order. Then a PDE invariant with respect to the infinitesimal generator (1.3) can be written as

$$
\mathscr{E}=\mathscr{E}\left(\left\{\mathscr{I}_{j}\right\}\right)=0, \quad j=0,1, \ldots
$$

Lie method is a well established technique to search for exact solutions of differential or difference equations of any type, integrable or non-integrable, linear or nonlinear. However, many equations may have no symmetries and there is no simple algorithm to prove the existence of symmetries other than looking for them. Moreover, the obtained solutions do not always fulfill the conditions imposed by the physical requests (boundary conditions, asymptotic behavior, etc.). So one looks for extension or modification of the construction which could overcome some of these problems. One looks for more symmetries,

- not always expressed in local form in terms of the dependent variable of the differential equations, - not satisfying all the properties of a Lie group but just providing solutions.

In the first class are the potential symmetries introduced by Bluman et al. $[4,8]$, the nonlocal symmetries by Vinogradov et al. $[13,24,26,31,34,39,40,46]$ while in the second one are the conditional symmetries [5, 19, 20,32,35]. 
The inverse problem, that is, to construct a differential equation with a prescribed set of Lie point symmetries has been discussed and used in many references (see for instance [6]), looking for models enjoying properties related to these symmetries. The basic idea is that by computing the invariants of the symmetries and then constructing the quantities written in terms of these invariants we can obtain equations that have prescribed symmetries and whose solutions have the given transformation properties, for example the Lorentz transformation.

A very important application in this field is the symmetry preserving discretization where we construct discretized equations which possess the same symmetries or a subgroup of the symmetry group of a given ODE or PDE [9,18,28-30]. The construction of the Ordinary Difference Equation $(\mathrm{O} \Delta \mathrm{E})$ or the Partial Difference Equation $(\mathrm{P} \Delta \mathrm{E})$ is obtained through the computation of discrete invariants of the symmetry group of the ODE or PDE or one of its subgroup looking for a function of these invariants which yields the ODE or the PDE in the continuous limit.

This paper is part of the work of extending the project of symmetry preserving discretization to the case of conditional symmetries. To do so we have to understand at first how to construct a differential equation possessing a given conditional symmetry starting from the infinitesimal generator. This is the content of this paper. The symmetry preserving discretization is left to future work.

As in other approaches to inverse problems, it is not evident that the equations we can find might have an immediate interest. This is the case, for example, of integrable equations associated to given spectral problems [12]. Here, as we will see in the examples, we have presented KdV-like equations which can be of interest in the description of shallow water waves in different physical contexts [37].

In Section 2 we will provide the theory behind the construction of the conditional symmetries clarifying in this way the difference between symmetries and conditional symmetries. Then in Section 3 we will verify the proposed construction in the case of the Boussinesq equation (3.1) and, in correspondence with its conditional symmetries, construct new conditionally invariant equations. Section 4 is devoted to the summary of the result, some concluding remarks and prospects of future works.

\section{What is a conditional symmetry?}

Conditional symmetries were introduced by Bluman and Cole with the name non-classical method [5] by adding an auxiliary first-order equation to (1.2), build up in terms of the coefficients of the infinitesimal generator $\hat{X}$, namely

$$
\mathscr{C}=\mathscr{C}\left(x, y, u, u_{x}, u_{y}\right)=\xi(x, y, u) u_{x}+\eta(x, y, u) u_{y}-\phi(x, y, u)=0,
$$

the infinitesimal symmetry generator (1.3) written in characteristic form [36] set equal to zero. Equation (2.1) is as yet unspecified and it will be determined together with the vector field $\hat{X}$, as it involves the same functions $\xi, \eta$ and $\phi$. I.e. we look for the simultaneous symmetry group of the overdetermined system of equations (1.2) and (2.1). It is easy to prove that (2.1) is invariant under the first prolongation of (1.3)

$$
\operatorname{pr} \hat{X} \mathscr{C}=-\left(\xi_{u} u_{x}+\eta_{u} u_{y}-\phi_{u}\right) \mathscr{C}
$$


without imposing any conditions on the functions $\xi, \eta$ and $\phi$. Consequently, we need just to apply the following invariance condition

$$
\left.\operatorname{pr} \hat{X} \mathscr{E}\right|_{\substack{\mathscr{E}=0 \\ \mathscr{C}=0}}=0
$$

Eq. (2.3) gives nonlinear determining equations for $\xi, \eta$ and $\phi$ which provide at the same time the classical and non-classical symmetries. In fact, as noted in [15], since all solutions of the classical determining equations necessarily satisfy the nonclassical determining equations (2.3), the solution set may be larger in the nonclassical case. As $\mathscr{C}=0$ appears in (2.3) as a condition imposed on the determining equations one has called the resulting symmetries conditional symmetries.

There are several works devoted to using the non-classical method to construct solutions of PDEs that are different from the ones obtained by classical method using the Lie point symmetries. Among them, let us cite as an example, [2, 17, 22, 23, 25, 38, 41]. Moreover we can find programs to compute them [3] and algorithms [14] for showing the existence of nontrivial nonclassical symmetries for given PDEs. In the case of integrable equations let us mention the works of Sergyeyev $[43,44]$ where he considered the classification of all $(1+1)$-dimensional evolution systems that admit a generalized (Lie-Bäcklund) vector field as a generalized conditional symmetry.

In this paper we want to look at the conditional symmetries from a different perspective. Given an infinitesimal group generator characterized by a vector field $\hat{X}$ for specific values of the functions $\xi, \eta$ and $\phi$, we want to construct equations $\mathscr{E}=0$ which have this symmetry as a conditional symmetry and not as a Lie point symmetry. Taking into account that an equation invariant under a given symmetry is written in terms of its invariants (1.6), a second order PDE invariant under a conditional symmetry will be given by

$$
\left.\mathscr{E}\left(\left\{\mathscr{I}_{j}\right\}\right)\right|_{\{\mathscr{C}=0\}}=0, \quad j=0,1, \ldots
$$

The constraint $\{\mathscr{C}=0\}$ in (2.4) is to be interpreted as the differential equation (2.1) and all of its differential consequences (see Section 3 for the details presented in the explicit examples).

The condition $\mathscr{C}=0$ and its differential consequences must not be used everywhere on the invariant equation to get (2.3) as, if we would do so, the global substitution of the condition and its consequences would turn the invariant PDE into an ODE in one of the independent variables with parametric dependence on the other.

\section{A series of examples including the Boussinesq equation}

The Boussinesq equation

$$
u_{y y}+u u_{x x}+\left(u_{x}\right)^{2}+u_{x x x x}=0,
$$

was introduced in 1871 by Boussinesq to describe the propagation of long waves in shallow water $[10,11]$ and it is of considerable physical and mathematical interest. It also arises in several other physical applications including one-dimensional nonlinear lattice waves [45, 47], vibrations in a nonlinear string [48], and ion sound waves in a plasma [42].

If $\eta$ in (1.3) is different from zero the resulting determining equations for conditional symmetries do not fix it and we can always put it equal to one. If $\eta=0$ and $\xi \neq 0$, we can put $\xi=1$ for the same reason. 
The conditional symmetries of the Boussinesq equation for $\eta \neq 0$ were obtained in [32], and in [16] by non group techniques. The case $\eta=0$ has been considered later and can be found in [15]. Moreover it is worthwhile to notice that the condition is the same if we consider $\hat{X}$ or $f(x, y, u) \hat{X}$, however the invariants in the two cases are different. So, for any $\hat{X}$ we can consider $f(x, y, u) \mathscr{C}=0$ as a condition.

In $[15,32]$ we find the following generators of the conditional symmetries for $(3.1)$ :

$$
\begin{aligned}
\hat{X}_{1} & =\partial_{y}+y \partial_{x}-2 y \partial_{u} \\
\hat{X}_{2} & =\partial_{y}-\frac{x}{y} \partial_{x}+\left(\frac{2}{y} u+\frac{6}{y^{3}} x^{2}\right) \partial_{u} \\
\hat{X}_{3} & =\partial_{y}+\left(-\frac{x}{y}+y^{4}\right) \partial_{x}+\left(\frac{2}{y} u+\frac{6}{y^{3}} x^{2}-2 y^{2} x-4 y^{7}\right) \partial_{u} \\
\hat{X}_{4} & =\partial_{y}+\left(\frac{x}{2 y}+y\right) \partial_{x}-\frac{1}{y}\left(u+2 x+4 y^{2}\right) \partial_{u} \\
\hat{X}_{5} & =\partial_{y}+\frac{1}{2} \frac{\dot{\wp}}{\wp}\left(x+\beta_{2} W\right) \partial_{x}-\left[\frac{\dot{\wp}}{\wp} u+3 \dot{\wp} x^{2}+\frac{\beta_{2}}{2}\left(\frac{1}{\wp}+12 \dot{\wp} W\right)\right. \\
& \left.+\frac{\beta_{2}^{2}}{2} W\left(\frac{1}{\wp}+6 \dot{\wp} W\right)\right] \partial_{u}, \quad W(y)=\int_{0}^{y} \frac{\wp(s)}{[\dot{\wp}(s)]^{2}} d s, \\
\hat{X}_{6} & =\partial_{x}+\left[\frac{2}{x+c_{0}} u+\frac{48}{\left(x+c_{0}\right)^{3}}\right] \partial_{u}, \\
\hat{X}_{7} & =\partial_{x}+\left[-2 x Q+c_{1} Q+c_{2} Q \int_{0}^{y} \frac{d s}{[Q(s)]^{2}}\right] \partial_{u},
\end{aligned}
$$

where $\wp$ is a special case of the Weierstrass elliptic function $\wp\left(y, g_{2}, g_{3}\right)$ [1] with $g_{2}=0$ satisfying the differential equation $\dot{\emptyset}^{2}=4 \wp^{3}-g_{3}, Q=\wp\left(y+c_{3}, 0, g_{3}\right)$ and $\beta_{2}, g_{3}$ and $c_{i}, i=0, \ldots, 3$ are arbitrary constants.

The generators $\hat{X}_{1}, \ldots, \hat{X}_{5}$ were obtained assuming $\eta=1$, thus are defined in (3.2-3.6) up to an arbitrary function $\eta(x, y, u)$ while $\hat{X}_{6}$ and $\hat{X}_{7}$ were obtained assuming $\eta=0$ and $\xi=1$, thus are defined in (3.7) and (3.8) up to an arbitrary function $\xi(x, y, u)$.

\subsection{Conditional invariant equations associated to $\hat{X}_{1}$}

For the infinitesimal generator $\hat{X}_{1}$ and its prolongation up to fourth order we obtain the following invariants:

$$
\begin{aligned}
\mathscr{I}_{0} & =-2 x+y^{2}, \quad \mathscr{I}_{1}=2 x+u, \quad \mathscr{I}_{2}=u_{x}, \quad \mathscr{I}_{3}=2 y+y u_{x}+u_{y}, \quad \mathscr{I}_{4}=u_{x x}, \\
\mathscr{I}_{5} & =y u_{x x}+u_{x y}, \quad \mathscr{I}_{6}=u_{y y}+2 y u_{x y}+2\left(y^{2}-x\right) u_{x x}, \quad \mathscr{I}_{7}=u_{x x x} \\
& \ldots, \mathscr{I}_{11}=u_{x x x x} .
\end{aligned}
$$

The condition is given by $\mathscr{I}_{3}=0$ i.e. $\mathscr{C}=2 y+y u_{x}+u_{y}=0$ and we can construct the Boussinesq equation in terms of the invariants (3.9). It is:

$$
\mathscr{I}_{1} \mathscr{I}_{4}+\mathscr{I}_{6}+\mathscr{I}_{11}+\left.\mathscr{I}_{2}^{2}\right|_{\mathscr{C}_{x}=0}=0
$$


As $\mathscr{C}_{x}=u_{x y}+y u_{x x}$ we have:

$$
\begin{aligned}
& u_{y y}+2 y\left(u_{x y}+y u_{x x}\right)+u u_{x x}+u_{x x x x}+\left.\left(u_{x}\right)^{2}\right|_{u_{x y}+y u_{x x}=0}= \\
& =u_{y y}+u u_{x x}+u_{x x x x}+\left(u_{x}\right)^{2}=0 .
\end{aligned}
$$

So, the Boussinesq equation (3.1) has the conditional symmetry given by $\hat{X}_{1}$.

Now, we want to construct an autonomous equation which have the conditional symmetry given by $\hat{X}_{1}$. Let us consider a different subset of the invariants (3.9)

$$
\mathscr{I}_{6}+\left.\mathscr{I}_{1} \mathscr{I}_{4}\right|_{\mathscr{C}_{x}=0}=0 \text {, i.e. }\left.\left[u_{y y}+2 y\left(u_{x y}+y u_{x x}\right)+u u_{x x}\right]\right|_{u_{x y}+y u_{x x}=0}=0 .
$$

So we we have:

$$
u u_{x x}+u_{y y}=0,
$$

a nonlinear Laplace equation which is a truncation of the Boussinesq equation.

To verify if effectively (3.12) has $\hat{X}_{1}$ as a conditional symmetry we compute its Lie point symmetries. They are

$$
\begin{aligned}
& \hat{Z}_{1}=\partial_{x} \\
& \hat{Z}_{2}=\partial_{y} \\
& \hat{Z}_{3}=x \partial_{x}+y \partial_{y} .
\end{aligned}
$$

As it does not exist a function $\eta(x, y, u)$ such that $\hat{Z}=\eta(x, y, u) \hat{X}_{1}$, for $\hat{Z}$ in (3.13), then (3.12) does have $\hat{X}_{1}$ as a conditional symmetry.

A KdV like non-autonomous equation which has the conditional symmetry given by $\hat{X}_{1}$ can be found by considering a different subset of the invariants (3.9), i.e.

$$
\mathscr{I}_{7}+\left.\left(\mathscr{I}_{1}+\mathscr{I}_{0}\right) \mathscr{I}_{2}\right|_{\mathscr{C}=0}=0 \text {, i.e. }\left.\left(u_{x x x}+u u_{x}+y^{2} u_{x}\right)\right|_{y u_{x}=-u_{y}-2 y}=0 .
$$

We get:

$$
u_{y}=\frac{1}{y}\left(u_{x x x}+u u_{x}\right)-2 y .
$$

To verify if effectively (3.15) has $\hat{X}_{1}$ as a conditional symmetry we compute its Lie point symmetries. They are

$$
\begin{aligned}
& \hat{Z}_{4}=\partial_{x} \\
& \hat{Z}_{3}=\ln y \partial_{x}-\partial_{u} \\
& \hat{Z}_{2}=\left[y^{2} \ln y+\frac{x}{3}-\frac{y^{2}}{6}\right] \partial_{x}+y \ln y \partial_{y}-\left[2 y^{2} \ln y+\frac{2 y^{2}}{3}-\frac{2 u}{3}\right] \partial_{u} \\
& \hat{Z}_{1}=y^{2} \partial_{x}+y \partial_{y}-2 y^{2} \partial_{u}=y \hat{X}_{1} .
\end{aligned}
$$

The invariants of $\hat{Z}_{1}$ are:

$$
\begin{aligned}
& I_{0}=2 x+u, \quad I_{1}=-2 x+y^{2}, \quad I_{2}=u_{x}, \quad I_{3}=y u_{y}+2 x u_{x}+4 x, \\
& I_{4}=u_{x x}, \quad I_{5}=2 x u_{x x}+y u_{x y}, \\
& I_{6}=y^{2} u_{y y}+4 x y u_{x y}+4 x^{2} u_{x x}+2 x u_{x}+4 x, \quad I_{7}=u_{x x x}, \ldots
\end{aligned}
$$


and (3.15) is given by $I_{0} I_{2}-I_{3}+I_{7}-2 I_{1}=0$. As $\hat{Z}_{1}=y \hat{X}_{1}, \hat{X}_{1}$ is a trivial conditional symmetry.

\subsection{Conditional invariant equations associated to $\hat{X}_{2}$}

For the infinitesimal generator $\hat{X}_{2}$ and its prolongation up to derivatives of fourth order we obtain the following invariants:

$$
\begin{aligned}
& \mathscr{I}_{0}=x y, \quad \mathscr{I}_{1}=x^{2} u+\frac{x^{4}}{y^{2}}, \quad \mathscr{I}_{2}=x^{3} u_{x}+2 \frac{x^{4}}{y^{2}}, \\
& \mathscr{I}_{3}=\frac{x^{2}}{y}\left(y u_{y}-x u_{x}-2 u-6 \frac{x^{2}}{y^{2}}\right), \quad \mathscr{I}_{4}=x^{4} u_{x x}+2 \frac{x^{4}}{y^{2}}, \\
& \mathscr{I}_{5}=\frac{x^{3}}{y}\left(y u_{x y}-x u_{x x}-3 u_{x}-12 \frac{x}{y^{2}}\right), \\
& \mathscr{I}_{6}=\frac{x^{2}}{y}\left(y u_{y y}-2 x u_{x y}+\frac{x^{2}}{y} u_{x x}-4 u_{y}+6 \frac{x}{y} u_{x}+6 \frac{u}{y}+42 \frac{x^{2}}{y^{3}}\right), \\
& \mathscr{I}_{7}=x^{5} u_{x x x}, \ldots, \mathscr{I}_{11}=x^{6} u_{x x x x} .
\end{aligned}
$$

The condition is given by $\mathscr{I}_{3}=0$ i.e. $\mathscr{C}=y u_{y}-x u_{x}-2 u-6 \frac{x^{2}}{y^{2}}=0$ and we can construct a nonlinear evolution PDE in terms of the invariants (3.18). It is:

$$
\mathscr{I}_{1} \mathscr{I}_{4}+\mathscr{I}_{11}+\mathscr{I}_{2}^{2}=x^{6}\left[u_{y y}+u u_{x x}+u_{x x x x}+\left(u_{x}\right)^{2}\right]
$$

when $\mathscr{C}=0$ and all its differential consequences.

A KdV like non-autonomous equation which may have the conditional symmetry given by $\hat{X}_{2}$ can be found by considering a different subset of the invariants (3.18), i.e.

$$
\mathscr{I}_{7}+\left.\mathscr{I}_{1} \mathscr{I}_{2}\right|_{\mathscr{C}=0}=0
$$

that is

$$
u_{y}+\frac{y}{x}\left(u_{x x x}+u u_{x}\right)-4 \frac{x^{2}}{y^{3}}=0
$$

Lie point symmetries of (3.21) are

$$
\begin{aligned}
& \hat{Z}_{1}=y \partial_{y}+\frac{1}{2} x \partial_{x}-u \partial_{u} \\
& \hat{Z}_{2}=\frac{1}{y^{5}}\left[\partial_{y}-\frac{x}{y} \partial_{x}+\left(\frac{2}{y} u+\frac{6}{y^{3}} x^{2}\right) \partial_{u}\right] \\
& \hat{Z}_{3}=y^{7} \partial_{y}+2 y^{6} x \partial_{x}-4 y^{4}\left(y^{2} u-3 x^{2}\right) \partial_{u}
\end{aligned}
$$

We observe that $\hat{Z}_{3}=\frac{1}{y^{5}} \hat{X}_{2}$. The invariants of $\hat{Z}_{3}$ are:

$$
\begin{aligned}
& I_{0}=x y, \quad I_{1}=x^{2}\left(u+\frac{x^{2}}{y^{2}}\right), \quad I_{2}=x^{3}\left(u_{x}+\frac{2 x}{y^{2}}\right), \\
& I_{3}=x^{7}\left(u_{y}+\frac{x}{y} u_{x}-\frac{2}{y} u-\frac{6 x^{2}}{y^{3}}\right), \quad I_{4}=x^{4}\left(u_{x x}+\frac{2}{y^{2}}\right), \ldots \\
& I_{7}=x^{5} u_{x x x},
\end{aligned}
$$


and (3.21) is given by $I_{3}+I_{0} I_{7}+I_{0} I_{1} I_{2}=0$. Thus the equation (3.21) is invariant under the vector field $\hat{Z}_{3}$ of (3.22) and $\hat{X}_{2}$ is a trivial conditional symmetry.

\subsection{Conditional invariant equations associated to $\hat{X}_{3}$}

For the infinitesimal generator $\hat{X}_{3}$ and its prolongation up to derivatives of fourth order we obtain the following invariants:

$$
\begin{aligned}
& \mathscr{I}_{0}=x y-\frac{1}{6} y^{6}, \quad \mathscr{I}_{1}=\frac{u}{y^{2}}+\frac{x^{2}}{y^{4}}-\frac{1}{3} x y+\frac{13}{18} y^{6}, \quad \mathscr{I}_{2}=\frac{u_{x}}{y^{3}}+2 \frac{x}{y^{5}}, \\
& \mathscr{I}_{3}=\frac{1}{y^{3}}\left[4 y^{8}+y^{5} u_{x}+2 y^{3} x+y u_{y}-x u_{x}-2 u-6 \frac{x^{2}}{y^{2}}\right], \quad \mathscr{I}_{4}=\frac{u_{x x}}{y^{4}}+2 \frac{1}{y^{6}}, \\
& \mathscr{I}_{7}=\frac{u_{3 x}}{y^{5}}, \ldots, \mathscr{I}_{11}=\frac{u_{x x x x}}{y^{6}}, \ldots .
\end{aligned}
$$

The condition is given by $\mathscr{I}_{3}=0$ i.e. $\mathscr{C}=4 y^{8}+y^{5} u_{x}+2 y^{3} x+y u_{y}-x u_{x}-2 u-6 \frac{x^{2}}{y^{2}}=0$ and we can construct a nonlinear evolution PDE in terms of the invariants (3.24), provided the condition is satisfied. It is:

$$
\mathscr{I}_{2}\left(\mathscr{I}_{2}-9\right)+\mathscr{I}_{11}+\mathscr{I}_{4}\left(\mathscr{I}_{1}-\frac{5}{3} \mathscr{I}_{0}\right)-36=\frac{1}{y^{6}}\left[u_{y y}+u u_{x x}+u_{x x x x}+\left(u_{x}\right)^{2}\right]
$$

when $\mathscr{C}=0$ and all its differential consequences.

Then, the Boussinesq equation (3.1) has the conditional symmetry given by the vector field $\hat{X}_{3}$.

A KdV like non-autonomous equation which may have the conditional symmetry given by $\hat{X}_{3}$ can be found by considering a different subset of the invariants (3.24), i.e.

$$
\left(\frac{637}{75} \mathscr{I}_{0}+\mathscr{I}_{1}\right) \mathscr{I}_{2}+\mathscr{I}_{7}-\left.\frac{8}{25} \mathscr{I}_{1}\right|_{\mathscr{C}=0}=0
$$

We get:

$$
x u_{y}+y\left[u_{x x x}+u u_{x}\right]=\frac{49}{75} y^{4} u-y^{4}\left(\frac{229}{25} x-\frac{52}{75} y^{5}\right) u_{x}-\frac{53}{3} x^{2} y^{2}+4 \frac{x^{3}}{y^{3}} .
$$

The equation (3.27) has no point symmetries. Then (3.27) is a conditionally invariant KdV-like equation.

\subsection{Conditional invariant equations associated to $\hat{X}_{6}$}

For the infinitesimal generator $\hat{X}_{6}$ with $c_{0}=0$ and its prolongation up to second order we obtain the following invariants:

$$
\begin{aligned}
& \mathscr{I}_{0}=y, \quad \mathscr{I}_{1}=\frac{1}{x^{4}}\left(12+x^{2} u\right), \quad \mathscr{I}_{2}=\frac{u_{y}}{x^{2}}, \\
& \mathscr{I}_{3}=\frac{1}{x^{5}}\left(x^{3} u_{x}-2 x^{2} u-48\right), \quad \mathscr{I}_{4}=\frac{u_{y y}}{x^{2}}, \ldots, \\
& \mathscr{I}_{10}=\frac{1}{x^{7}}\left(-1440-24 x^{2} u+18 x^{3} u_{x}+x^{5} u_{x x x}-6 x^{4} u_{x x}\right) .
\end{aligned}
$$


The condition is given by $\mathscr{I}_{3}=0$ i.e. $\mathscr{C}=u_{x}-\frac{2 u}{x}-\frac{48}{x^{3}}=0$ and we can construct a nonlinear evolution PDE in terms of the invariants (3.28). It is:

$$
6 \mathscr{I}_{1}^{2}+\mathscr{I}_{4}=\frac{1}{x^{2}}\left[u_{y y}+u u_{x}+u_{x x x x}+\left(u_{x}\right)^{2}\right]
$$

when $\mathscr{C}=0$ and all its differential consequences.

Then, the Boussinesq equation (3.1) has the conditional symmetry given by $\hat{X}_{6}$.

A KdV like non-autonomous equation which may have the conditional symmetry given by $\hat{X}_{6}$ can be found by considering a different subset of the invariants of (3.28), i.e.

$$
\mathscr{I}_{3}^{2}+\mathscr{I}_{2}+\left.\mathscr{I}_{10}\right|_{\mathscr{C}_{\mathscr{C}=0}=0}=0
$$

Eq. (3.30) reads:

$$
u_{y}+u_{x x x}-4 \frac{u u_{x}}{x^{3}}+8 \frac{u^{2}}{x^{4}}+192 \frac{u}{x^{6}}-\frac{288}{x^{5}}=0
$$

Equation (3.31) has only Lie point symmetry $\hat{Z}_{1}=\partial_{y}$.

$$
\hat{Z}_{1}=\partial_{y}
$$

So, (3.31) is a conditionally invariant equation.

\subsection{Conditional invariant equation associated to $\hat{Y}=\partial_{y}+\frac{x}{2 y} \partial_{x}-\frac{1}{y} \partial_{u}$}

The generator $\hat{Y}=\partial_{y}+\frac{x}{2 y} \partial_{x}-\frac{1}{y} \partial_{u}$ was introduced by Momoniat [33] to describe the nonclassical (conditional) symmetries of the Frank-Kamenetskii partial differential equation

$$
\frac{\partial u}{\partial y}=\frac{1}{x} \frac{\partial}{\partial x}\left(x \frac{\partial u}{\partial x}\right)+e^{u}
$$

modeling a thermal explosion in a cylindrical vessel. In [33] the obtained symmetry for (3.33) was shown to correspond to a classical symmetry.

For the infinitesimal generator $\hat{Y}$ and its prolongation up to third order we obtain the following invariants:

$$
\begin{aligned}
& \mathscr{I}_{0}=\frac{y}{x^{2}}, \quad \mathscr{I}_{1}=2 \ln x+u, \quad \mathscr{I}_{2}=x u_{x}, \quad \mathscr{I}_{3}=\frac{1}{2 y}\left(2+x u_{x}+2 y u_{y}\right), \\
& \mathscr{I}_{4}=x^{2} u_{x x}, \ldots, \quad \mathscr{I}_{6}=u_{y y}+\frac{x}{y} u_{x y}+\frac{1}{4} \frac{x^{2}}{y^{2}} u_{x x}-\frac{1}{4} \frac{x}{y^{2}} u_{x}-\frac{1}{y^{2}}, \\
& \mathscr{I}_{7}=x^{3} u_{x x x} .
\end{aligned}
$$

The condition is given by $\mathscr{I}_{3}=0$ i.e. $\mathscr{C}=2+x u_{x}+2 y u_{y}=0$ and we can construct, apart from the Frank-Kamenetskii partial differential equation (3.33), a nonlinear KdV like evolution PDE in 
terms of the invariants (3.34). It is:

$$
\mathscr{I}_{0} e^{\mathscr{I}_{1}}+\mathscr{I}_{0} \mathscr{I}_{7}+1+\left.\frac{1}{2} \mathscr{I}_{2}\right|_{\mathscr{C}=0}=0, \quad \text { i.e. } \quad u_{y}=x u_{x x x}+e^{u}
$$

i.e. a nonlinear dispersive non-autonomous KdV like equation. Lie point symmetries of (3.35) are

$$
\begin{aligned}
& \hat{Z}_{1}=\partial_{y} \\
& \hat{Z}_{2}=y \partial_{y}+\frac{1}{2} x \partial_{x}-\partial_{u}
\end{aligned}
$$

We observe that $\hat{Z}_{2}=y \hat{Y}$. The invariants of $\hat{Z}_{2}$ are:

$$
I_{0}=\frac{y}{x^{2}}, I_{1}=2 \ln x+u, I_{2}=x u_{x}, I_{3}=x^{2} u_{y}, \ldots, I_{7}=x^{3} u_{x x x},
$$

and (3.35) is given by $I_{3}-I_{7}-\exp \left(I_{1}\right)=0$. Thus the equation (3.35) is invariant under $\hat{Z}_{2}$ and $\hat{Y}$ is, in this sense, a trivial conditional symmetry.

For this infinitesimal generator we can construct a conditionally invariant wave equation too,

$$
\mathscr{I}_{6}+\left.\mathscr{I}_{0}^{-2} e^{\mathscr{I}_{1}}\right|_{\mathscr{C}_{\mathscr{C}=0}=0}=0 \text {, i.e. } y^{2} u_{y y}-\frac{1}{4} x^{2} u_{x x}-\frac{3}{4} x u_{x}-1+x^{6} e^{u}=0,
$$

a nonlinear non autonomous wave equation which has as Lie point symmetries of infinitesimal generators

$$
\begin{aligned}
& \hat{W}_{1}=y \partial_{y} \\
& \hat{W}_{2}=x \partial_{x}-6 \partial_{u} \\
& \hat{W}_{3}=\frac{1}{x y} \partial_{x}+\frac{2}{x^{2}} \partial_{y}-\frac{2}{y x^{2}} \partial_{u}=\frac{2}{x^{2}} \hat{Y} .
\end{aligned}
$$

In correspondance with $\hat{W}_{3}$, after some nontrivial calculations, we get the following invariants

$$
\begin{aligned}
& J_{0}=\frac{y}{x^{2}}, J_{1}=2 \ln x+u, J_{2}=\frac{1}{4 y x^{2}}\left(2 y u_{y}+x u_{x}+2\right), \\
& J_{3}=\frac{x^{2}}{4 y}\left(2 y u_{y}-x u_{x}-2\right), J_{4}=\frac{1}{8 y^{2}}\left(4 y^{2} u_{y y}-x^{2} u_{x x}-x u_{x}+4 y u_{y}\right) .
\end{aligned}
$$

Then (3.38) is written in terms of the invariants (3.40) as $J_{4}-J_{2} J_{0}^{-1}+\frac{1}{2} e^{J_{1}} J_{0}^{-2}=0$. So, also the equation (3.38) has in $\hat{Y}$ a trivial conditional symmetry.

\section{Conclusions}

In this article we presented a construction of nonlinear PDE's having given conditional symmetries. They are obtained starting from the invariants of the symmetry and imposing the extra condition given by equating to zero the characteristic and its differential consequences. We showed in the case of the Boussinesq equation that effectively the construction proposed works and allows to produce other conditionally invariant nonlinear equations $(3.12,3.15,3.21,3.27,3.31,3.35,3.38)$. Equations $(3.15,3.21,3.27,3.31,3.35)$ are non-autonomous $\mathrm{KdV}$-like equations and it is well known that the $\mathrm{KdV}$ equation has no non-singular conditional symmetries [16]. However, not all obtained equations are conditionally invariant even if we constructed them in such a way. The obtained equations can 
still have the generator $\hat{X}$ as a point symmetry due to the arbitrary multiplicative factor $\eta(x, y, u)$ or $\xi(x, y, u)$ under which the condition is defined. This is what happens in cases of the KdV-like equations $(3.15,3.21,3.35)$ and of the nonlinear wave equation (3.38).

An important point not touched in this work but on which we are presently working is understanding a priori if this technique can be used to characterize the class of nonlinear PDEs that allows conditional symmetries (see the paper by Kunzinger and Popovych [27] for results on this problem). Why most of the KdV like equation we have constructed are not conditionally invariant?

Moreover work is also in progress on solving by symmetry reduction the obtained conditionally invariant KdV like equations and on the construction of conditional symmetry preserving discretizations of the Boussinesq equation.

\section{Acknowledgments}

DL has been supported by INFN IS-CSN4 Mathematical Methods of Nonlinear Physics. DL thanks ZT and the SUNY Polytechnic Institute for their warm hospitality at Utica when this work was started. DL thanks the Departamento de Física Téorica of the Universidad Complutense de Madrid for its hospitality. MAR was supported by the Spanish MINECO under project FIS 2015-63966-P. D. Nedza, summer student of ZT, contributed to the verification of some of the computations.

\section{References}

[1] M. Abramowitz and I.A. Stegun, Handbook of Mathematical Functions, Dover, New York, 1972.

[2] D.J. Arrigo, B.P. Ashley, S.J. Bloomberg and T.W. Deatherage, Nonclassical Symmetries of a Nonlinear Diffusion-Convection/Wave Equation and Equivalents Systems, Symmetry 8 (2016), 140.

[3] N. Bîlă and J. Niesen, On a new procedure for finding nonclassical symmetries, J. Symb. Comp., 38, (2004), 1523-1533.

[4] G.W. Bluman, Use and construction of potential symmetries, Math. Comp. Modelling, 18 (1993), 1-14.

[5] G.W. Bluman and J.D. Cole, The general similarity solutions of the heat equation J. Math. Mech., 18 (1969), 1025-1042.

[6] G.W. Bluman, J.D. Cole, Similarity methods of differential equations, Springer, New York, 1974.

[7] G.W. Bluman and S. Kumei, Symmetries of Differential Equations, Springer-Verlag, New York, 2002.

[8] G.W. Bluman, S. Kumei, and G.J. Reid, New classes of symmetries for partial differential equations, $J$. Math. Phys., 29 (1988), 806-811.

[9] A. Bourlioux, C. Cyr-Gagnon, and P. Winternitz, Difference schemes with point symmetries and their numerical tests, J. Phys. A: Math. Gen., 39 (2006), 6877-6896.

[10] J. Boussinesq, Théorie de l'intumescence liquide appelée onde solitaire ou de translation se propageant dans un canal rectangulaire, Comptes Rendus, 72 (1871), 755-759.

[11] J. Boussinesq, Théorie des ondes et des remous qui se propagent le long d'un canal rectangulaire horizontal, en communiquant au liquide contenu dans ce canal des vitesses sensiblement pareilles de la surface au fond, J. Math. Pures Appl., 7 (1872), 55-108.

[12] M. Bruschi, D. Levi and O. Ragnisco, Nonlinear evolution equations solvable by the inverse spectral transform associated to the matrix Schroedinger equation of rank 4, Il Nuovo Cimento, B43 (1978), 251-270.

[13] D. Catalano Ferraioli, Nonlocal aspects of $\lambda$-symmetries and ODEs reduction, J. Phys A: Math. Theor., 40 (2007), 5479-5489.

[14] Chaolu Temuer and G. Bluman, An algorithmic method for showing existence of nontrivial nonclassical symmetries of partial differential equations without solving determining equations, J. Math. Anal. Appl., 411 (2014), 281-296.

[15] P.A. Clarkson, Nonclassical symmetry reductions of the Boussinesq equation, Chaos Solitons Fractals, 5 (1995), 2261-2301. 
[16] P.A. Clarkson and M.D. Kruskal, New similarity reductions of the Boussinesq equation, J. Math. Phys., 30 (1989), 2201-2213.

[17] P.A. Clarkson and E.L. Mansfield, Symmetry Reductions and Exact Solutions of Shallow Water Wave Equations, Acta Appl. Math., 39 (1995), 245-276.

[18] V.A. Dorodnitsyn, Applications of Lie Groups to Difference Equations, CRC Press, Boca Raton, Fla. (2011).

[19] W.I. Fushchich, Conditional symmetry of the equations of nonlinear mathematical physics, Ukr. Math. J., 43 (1991), 1350-1364.

[20] W.I. Fushchich and R.Z. Zhdanov, Conditional symmetry and reduction of partial differential equations, Ukr. Math. J., 44 (1993), 875-886

[21] G. Galilei, Il Saggiatore: Nel Quale Con Bilancia Esquisita E Giusta Si Ponderano Le Cose Contenute Nella Libra Astronomica E Filosofica Di Lotario Sarsi Sigensano, Scritto in Forma Di Lettera All'Illustr. Et Rever. Mons. D. Virginio Cesarini, G. Mascardi, Roma 1623.

[22] R.K. Gupta and M. Singh, Nonclassical symmetries and similarity solutions of variable coefficient coupled KdV system using compatibility method, Nonlinear Dyn., 87 (2017), 1543-1552.

[23] M.S. Hashemi and M.C. Nucci, Nonclassical symmetries for a class of reaction-diffusion equations: the method of heir-equations, J. Nonlinear Math. Phys., 20 (2013), 44-60.

[24] R. Hernández-Heredero and E.G. Reyes, Nonlocal symmetries and a Darboux transformation for the Camassa-Holm equation, J. Phys. A: Math. Theor., 42 (2009), 182002.

[25] L. Ji, C.Z. Qu, and S. Shen, Conditional Lie-Backlund Symmetry of Evolution System and Application for Reaction-Diffusion System, Stud. Appl. Math., 133 (2014), 118-149.

[26] I.S. Krasil'shchik and A.M. Vinogradov, Nonlocal trends in the geometry of differential equations: symmetries, conservation laws, and Bäcklund transformations. Symmetries of partial differential equations, Part I, Acta Appl. Math., 15 (1989), 161-209.

[27] M. Kuzinger and R. Popovych, Is a nonclassical symmetry a symmetry? in Proceedings of 4th Workshop Group Analysis of Differential Equations and Integrable Systems (26-30 October 2008, Protaras, Cyprus), (2009), 107-120.

[28] D. Levi, L. Martina, and P. Winternitz, Lie-point symmetries of the discrete Liouville equation, J. Phys. A: Math. Theor., 48 (2015), 025204.

[29] D. Levi, L. Martina, and P. Winternitz, Structure preserving discretizations of the Liouville equation and their numerical tests, SIGMA, 11 (2015), 080.

[30] D. Levi, L. Martina, and P. Winternitz, Conformally invariant elliptic Liouville equation and its symmetry preserving discretization, Theor. Math. Phys., 196 (2018), 1307-1319.

[31] D. Levi, M.C. Nucci and M.A. Rodríguez, $\lambda$ symmetries for the reduction of continuous and discrete equations, Acta Appl. Math., 122 (2012), 311-321.

[32] D. Levi and P. Winternitz, Nonclassical symmetry reduction: Example of the Boussinesq equation, $J$. Phys. A: Math. Gen., 22 (1989), 2915-2924.

[33] E. Momoniat, A thermal explosion in a cylindrical vessel: A non-classical symmetry approach, Int. J. Mod. Phys. B23 (2009), 3089-3099.

[34] C. Muriel, J.L. Romero, New methods of reduction for ordinary differential equations, IMA J. Appl. Math. 66 (2001), 111-125.

[35] M.C. Nucci, P.A. Clarkson, The nonclassical method is more general than the direct method for symmetry reductions. An example of the Fitzhugh-Nagumo equation, Phys. Lett. A 164 (1992), 49-56.

[36] P.J. Olver Applications of Lie Groups to Differential Equations, Springer-Verlag, New York, 1993.

[37] P.J. Olver, and E.M. Vorob'ev, Nonclassical and conditional symmetries, in: CRC Handbook of Lie Group Analysis of Differential Equations, vol. 3, N.H. Ibragimov, ed., CRC Press, Boca Raton, Fl., 1996, 291-328.

[38] R.O. Popovych, N.M. Ivanova and O.O. Vaneeva, Potential nonclassical symmetries and solutions of fast diffusion equation, Phys. Lett. A, 362 (2007), 166-173.

[39] E.G. Reyes, Nonlocal symmetries and the Kaup-Kupershmidt equation, J. Math. Phys., 46 (2005), 073507. 
[40] E.G. Reyes, On nonlocal symmetries of some shallow water equations, J. Phys. A: Math. Theor., 40 (2007), 4467-4476.

[41] P.M.M. Rocha, F.C. Khannab, T.M. Rocha Filhoa and A.E. Santana, Non-classical symmetries and invariant solutions of non-linear Dirac equations Commun. Nonlinear Sci. Num. Simul., 26 (2015), 201-210.

[42] A.C. Scott, in Bäcklund Transformations, Lecture Notes in Mathematics, Vol. 515, edited by R.M. Miura, Springer, Berlin, (1975), 80-105.

[43] A. Sergyeyev, Constructing conditionally integrable evolution systems in (1+1) dimensions: a generalization of invariant modules approach, J. Phys. A: Math. Gen., 35 (2002), 7653-7660.

[44] A. Sergyeyev, On the classification of conditionally integrable evolution systems in $(1+1)$ dimensions, J. Math. Sci., 136 (2006), 4392-4400.

[45] M. Toda, Studies of a nonlinear lattice, Phys. Rep., 18 (1975), 1-125.

[46] A.M. Vinogradov and I.S. Krasil'shchik, A method of calculating higher symmetries of nonlinear evolutionary equations, and nonlocal symmetries. (Russian) Dokl. Akad. Nauk SSSR, 253 (1980), 1289-1293.

[47] N.J. Zabusky, A Synergetic Approach to Problems of Nonlinear Dispersive Wave Propagation and Interaction, in Nonlinear Partial Differential Equations, edited by W.F. Ames, Academic Press, New York, (1967), 233-258.

[48] V.E. Zakharov, On stochastization of one-dimensional chains of nonlinear oscillations Sov. Phys. JETP, 38 (1974), 108. 